\title{
"LAS EDADES EXTREMAS COMO FACTOR DE RIESGO EN LA PRESENCIA DE PREECLAMPSIA SEVERA EN EL HOSPITAL REGIONAL DOCENTE MATERNO INFANTIL EL CARMEN"
}

\section{"EXTREME AGES AS A RISK FACTOR IN SEVERE PREECLAMPSIA HRDMI EL CARMEN"}

\author{
Manuel M. Adrián Acosta ${ }^{1}$
}

\section{RESUMEN}

Se han revisado las historias clínicas materno perinatales del Hospital Regional Docente Materno Infantil "El Carmen", extraídas a través del SIP 200 , las cuales fueron procesadas de forma computarizada, utilizando los números absolutos y los porcentajes. En la evaluación de las Historias clínicas se encontró un total de 302 gestantes menores de 18 años y 253 gestantes mayores de 35 años, de las cuales 27 menores de 18 años y 29 mayores de 35 años presentaron Pre eclampsia Severa.

\section{ABSTRACT}

The clinical maternal perinatal stories from the Hospital Regional Docente Materno Infantil "El Carmen" have been reviewed, extracted through the SIP 200, which were processed in computerized form, using the absolute numbers and percentages. In the evaluation of the medical records it was found a total of 302 pregnant under the age of 18 and 253 pregnant over 35, of which 27 under the age of 18 and 29 older than 35 years old had severe Preeclampsia.

\section{INTRODUCCIÓN}

La Pre-eclampsia (PEC) es definida como el incremento de la presión arterial acompañada de edema, proteinuria 0 ambas que ocurre después de la 20a semana de gestación.

Cualquiera de los siguientes criterios son suficientes para el diagnóstico de Hipertensión: Aumento de la presión sistólica en 30 mkg o mayor, Aumento de la presión diastólica en 15 mkg o mayor. (1), TAM (Tensión Arterial Media) mayor a 105 mkg 0 incremento de 20 mkg en 2 determinaciones. (2)

En la epidemiologia la Edad más frecuente es en menores de 18 años y mayores de 35(2), aunque algunos estudios demuestran que el riesgo se puede observar en embarazos en menores de 21 años (1). La clase social, raza, nivel socioeconómico: contrariamente a lo que se creía no predisponen a padecer PEC con más frecuencia. En la Paridad, las primigestas tienen más posibilidades de enfermedad hipertensiva del embarazo, son 6 a 8 veces más susceptibles que las multíparas.(2) El riesgo de PEC es generalmente inferior en segundos embarazos que en primeros, pero no si la madre tiene un nuevo compañero para el segundo embarazo. Una explicación es que reducen el riesgo con la exposición repetida maternal y la adaptación a antígenos específicos del mismo compañero. Sin embargo la diferencia en el riesgo podría en cambio ser explicado por el intervalo entre nacimientos. Un intervalo de internacimiento más largo puede ser asociado tanto con un cambio de compañero como con un riesgo más alto de PEC. (4)

\section{MATERIAL Y METODOS}

\section{METODO DE INVESTIGACION:}

El trabajo de investigación se mantiene en un marco de estudio retrospectivo, analítico y de corte transversal.

1 MC. Profesor Auxiliar de Medicina, UNCP, Gineco Obstetra, Hospital Docente materno infantil "El Carmen". 


\section{DISEÑO METODOLOGICO}

\section{a) POBLACION Y MUESTRA:}

La población del presente estudio de investigación está constituido por las gestantes que acuden al Servicio de Emergencias del hospital Regional Docente Materno Infantil "El Carmen".

El total en la toma de datos de las pacientes que fueron atendidas en el Servicio de Emergencia de 3500 pacientes, dentro de ellas fueron 555 en edades extremas, presentando diagnóstico de Pre eclampsia severa un total de 56 , teniendo en el grupo de menores de 18 años 27 y las mayores de 35 años son 29.

\section{RESULTADOS Y DISCUSION}

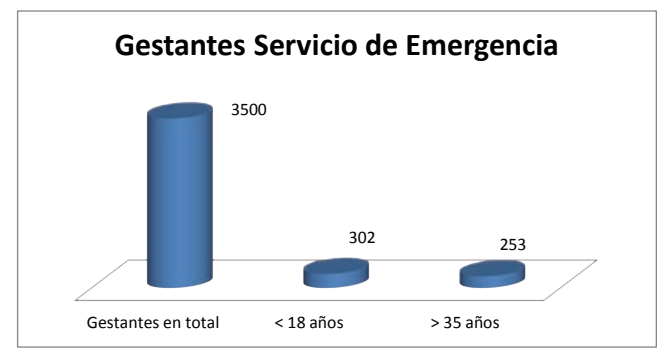

\section{Gestantes con Preeclampsia Severa}

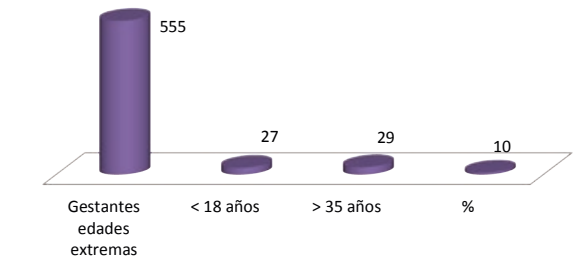

Encontrándose que menores de 18 años 302 y mayores de 35 años 253. De este universo 555 son de edades extremas.

De este total de gestantes en edades extremas para menores de 18 años se encontró 27 casos que corresponde a $4.8 \%$ y en mayores de 35 años 29 casos que corresponde a $5.2 \%$.

\section{DISCUSION}

En la revisión de historias clínicas en la base de datos del SIP 2000 del Hospital Regional Docente Materno Infantil "El Carmen" se ha encontrado que del total el $15.9 \%$ corresponde a gestantes en edades extremas de las cuales el $8.6 \%$ corresponde a menores de 18 años y mayores de 35 años son el $7.2 \%$ de lo que se puede ver que hay una tendencia al incremento de gestantes menores de 18 años.

En las pacientes menores de 18 años se encuentra que el $4.8 \%$ presentaron casos de pre eclampsia severa y en las mayores de 35 fue el $5.2 \%$.

Encontrando un $10 \%$ del total de gestantes en estas edades.

\section{CONCLUSIONES}

De lo revisado se puede ver que la complicación de pre eclampsia severa en las edades extremas es aproximadamente del 10\%.

Según el trabajo la tendencia es que esta se presente en mayores de 35 años con $5.2 \%$

Recomendamos que se haga un buen tamizaje en gestantes mayores de 20 semanas priorizando a las pacientes en edades extremas menores de $18 \mathrm{y}$ mayores de 35 años

\section{REFERENCIAS BIBLIOGRÁFICAS}

1) Fernández Contreras R, Gómez Llambi H, Ferrarotti F, Lorge F. Extraido de "Guía para el manejo de la Hipertensión Arterial". Instituto de Investigaciones Cardiológicas - Facultad de Medicina -UBA.2000.

2) García Santos fJ, Costales Badillo CA, Jimeno JM. Fisiopatología y factores etiopatogénicos de la hipertensión arterial en el embarazo. Revisión de la Literatura. Hospital clínico San Carlos de Madrid. Cátedra de Obstetricia y Ginecología (Prof. M. Escudero Fernández). Toko -Ginecología Práctica Mayo 2000; Número 645 p.194 -212.

3) Lain KY, Wilson JW, Crombleholme WR, Ness RB, Roberts JM. Smoking during pregnancy is associated with alterations in markers of endothelial function. Am J ObstetGynecol 2003 0ct; 189(4):1196-201. 
4) Skjaerven R, Wilcox AJ, Lie RT .The Interval between pregnancies and the Risk of Preeclampsia. New England Journal Medical January 3, 2002 Number 1; Volume 346:33-38.

5) [ University of Virginia Health System ]. "La Hipertensión inducida por el Embarazo (HIE) "(en español). El Embarazo de Alto Riesgo. Consultado el 20 de diciembre del 2007.

6) A b c Drife J0, Magowan (eds). Clinical Obstetrics and Gynaecology, chapter 39, pp 367-370. ISBN 0-7020-1775-2.

7) Robbins and Cotran, Phatological Basis of Disease, 7 ht ed.

8) A b c Mattew Warden; Brian Euerle (7 de Mayo de 2005) . "Preeclamsia (Toxemia of Pregnancy)" (en inglés). Medicine - Obstetrics /ginecology. consultado el 20 de diciembre de 2007.

9) Hjartardottir S, Leifsson BG, Geirsson RT, Steinthorsdottir V. (2004) "Paternity change and the recurrence risk in familial hypertensive disorder in pregnancy. " Hypertens Pregnancy 2004; 23(2): 219 -25.PMID 15369654.
10) BLANCO, Mario, VASQUEZ, Manuel, TRIAS, Yalitze et al. Efecto de Metoclopramida en Mujeres Hipertensas en puerperio Inmediato .AVFT. [online]. Jan .2000, Vol .19, no.1 [citado 20 December 2007], p.62-64. Disponible en la World Wide web :[4] .ISSN 0798-0264.

11) Douglas K, Redman C (1994). "Eclampsia in the united Kingdom“. BMJ 309 (6966): PP.1395-400. PIMD 7819845.

12) [Medline Plus] (noviembre de 2008). "Preeclampsia raises risk of epilepsy in offpring "(en español) Enciclopedia Medica en español .consultado el 12 de noviembre del 2008.

13) PACHECO ROMERO, José - Manual de Obstetricia 6ta .Edición mayo 2011.

14) Guías de Práctica Clínica para la atención de emergencias obstétricas según nivel de capacidad resolutiva. Ministerio de Salud. 2007. 